\title{
Modelo bayesiano para el estudio de la enfermedad del dengue en el departamento de Atlántico, Colombia, años 2010 a 2013
}

\author{
BAYESIAN MODEL FOR THE STUDY OF DENGUE DISEASE IN THE \\ DEPARTMENT OF ATLÁNTICO COLOMBIA YEARS 2010 TO 2013 \\ MODELO BAYESIANO PARA O ESTUDO DA DOENÇA DO DENGUE NO \\ DEPARTAMENTO DE ATLÁNTICO, COLÔMBIA, ANOS 2010 A 2013
}

Recepción:

19 de septiembre de 2016

Evaluación:

ANGIE ANDREA Montoya González ${ }^{1}$ FABIÁN GABRIEL Ortiz Beltrán ${ }^{2}$ LUIS FERNANDO Santa Guzmán ${ }^{3}$

22 de junio de 2017

Aprobación:

12 de julio de 2017

Para citar este artículo: Montoya González, A. A., Ortiz Beltrán, F. G. y Santa Guzmán, L.F. (2017). Modelo bayesiano para el estudio de la enfermedad del dengue en el departamento de Atlántico, Colombia, años 2010 a 2013. Perspectiva Geográfica, 22(2),85-104 doi: 10.19053/01233769.7603

\section{Resumen}

El propósito en este artículo es estudiar la relación entre los casos de dengue y las variables dadas por los datos sociales, geográficos y económicos de los 23 municipios del departamento del Atlántico, Colombia, mediante el uso de modelos espaciales completamente bayesianos para el período 2010 a 2013. Se analizaron 7786 casos de dengue presentados en los cuatro años de estudio, en los que se encontró que los

1 Ingeniera catastral y geodesta en formación de la Universidad Distrital Francisco José de Caldas.anamontoyag@correo.udistrital.edu.co

2 Ingeniero catastral y geodesta en formación de la Universidad Distrital Francisco José de Caldas. fgortizb@correo.udistrital.edu.co

3 Ingeniero catastral y geodesta, estadista y magíster en Geomática. Doctorante en Geoinformática de la Universidad Nova de Lisboa. Ifsantag@unal.edu.co 
municipios más afectados por cantidad de población fueron Tubará, Candelaria, Puerto Colombia, Baranoa, Polonuevo y Ponedera. Se escogió el mejor modelo por cada año de estudio, basados en el Criterio de Información de Desviación (DIC), se encontró que las variables afines a las características sociales presentes en las viviendas y el crecimiento desordenado del casco urbano fueron las que mayor influencia tuvieron en el aumento del número de casos de dengue. El modelo bayesiano permitió identificar la relación del dengue con factores fuera del sector de salud estableciendo áreas de mayor riesgo de enfermedad.

Palabras clave: Atlántico, Colombia, dengue, epidemiología, estadística espacial, modelo bayesiano.

\section{Abstract}

This papers aims to study the relationship between dengue cases and the variables given by the social, geographic and economic data of the 23 municipalities of the department of Atlantico, Colombia, through the use of completely. It was possible to analyze 7.786 cases of dengue presented in the four years of study, finding that the municipalities most affected by amount of population were Tubará, Candelaria, Puerto Colombia, Baranoa, Polonuevo and Ponedera. The best model was chosen for each year of study, based on the Deviation Information Criterion (DIC), it was found that the variables related to the social characteristics present in the dwellings and the disordered growth of the urban center were those that had the greatest influence In the increase of the number of dengue cases. The Bayesian model allowed the identification of the relationship of dengue with factors outside the health sector, establishing areas of higher risk of disease.

Keywords: Atlantico, Colombia, dengue, epidemiology, spatial statistics, Bayesian model.

\section{Resumo}

O objetivo deste artigo é estudar a relação entre casos de dengue e as variáveis dadas pelos dados sociais, geográficos y econômicos dos 23 municípios do departamento de Atlántico, Colômbia, usando modelos espaciais completamente bayesianos para o período 2010 a 2013. Analisaram-se 7786 casos de dengue apresentados nos quatro anos de estudo, que constatou que os municípios mais afetados pelo tamanho da população foram Tubará, Candelaria, Puerto Colombia, Baranoa, Polonuevo e Ponedera. Se escolho o melhor modelo para cada ano de estudo, baseado no critério de Informação 
de Desvio (DIC), verificou-se que as variáveis relacionadas com características sociais presentes na habitação e o crescimento desordenado do casco urbano foram a maior influência no aumento do número de casos de dengue. O modelo bayesiano permitiu identificar a relação do dengue com fatores externos ao setor saúde, estabelecendo áreas de maior risco de doença.

Palavras chave: Atlantico, Colômbia, dengue, epidemiologia, estatísticas espaciais, modelo bayesiano.

\section{Introducción}

El dengue ha sido calificado por la Organización Mundial de la Salud (OMS) como "la enfermedad transmitida por mosquitos más importante del mundo", ya que afecta principalmente a continentes como Asia, África y América Latina, debido a la variabilidad climática intra-anual (Echavarria y Quintero, 2012). Existen diferentes factores interrelacionados que favorecen la propagación del dengue, entre ellos factores geográficos determinantes del clima, algunos de tipo socioeconómicos como la urbanización no planificada, las condiciones y calidad de vida de la población y ciertos factores culturales como la propagación de depósitos de agua. Es por esto que se estima anualmente en el mundo la infección de 390 millones de personas (Bhatt et al., 2013).

Diferentes modelos han surgido como herramientas de apoyo en materia de salud pública, por ende, las zonas más afectadas por el vector (Asia, África y Latinoamérica) han sido fuente de diferentes estudios como apoyo a las decisiones administrativas. Un modelo de redes neuronales artificiales refleja que factores como la temperatura media, la humedad relativa y la precipitación total son los parámetros de más influencia para la predicción del número de casos de dengue en algunos países asiáticos (Aburas, Cetiner y Sari, 2010). Un modelo temporal es el método aplicado en Tailandia como la medida de monitoreo y predicción de los casos de dengue, sus resultados son satisfactorios, ya que permiten comparar largos periodos de tiempo e identificar la frecuencia de la epidemia (Wongkoon, Jaroensutasinee y Jaroensutasinee, 2012). El modelo temporal resulta útil también en Malasia, donde la dependencia temporal de las variables climáticas en las series de tiempo favorecen la predicción de casos de dengue (Dom, Hassan, Latif e Ismail, 2013).

En Brasil, un país afectado gravemente por el virus, se realizó un modelo bayesiano de los casos de dengue y su relación con variables como el alfabetismo, ingresos, servicio de agua y recolección de basuras, presentados en la unidad federal Espíritu Santo, en el año 2010; con el modelo, se permite minimizar la varianza de los estimadores en lugares donde la población es pequeña e incluye los factores económicos, sociales y geográficos de cada región (Honorato et al., 2014). El modelo bayesiano aplicado para el área metropolitana de Maracay, Venezuela, revela la importancia de los indicadores socioeconómicos, su consideración proporciona mejores ajustes de modelo y sugiere que la intervención en estos puede disminuir el riesgo en contraer la enfermedad. A diferencia de los modelos empleados en el continente asiático, donde la variación climática en periodos de tiempo son herramientas satisfactorias para la predicción de casos de dengue, en Latinoamérica, el modelo 
bayesiano muestra que los casos de dengue no están determinados únicamente por las variables climáticas, sino por las condiciones socio-económicas (Monsalve, Rubio-Palis y Pérez, 2010).

Para Colombia, el dengue se ha convertido en un problema prioritario debido a que en el país circulan simultáneamente los cuatro serotipos causantes de la enfermedad, (DENV1, DENV2, DENV3 y DENV4). El mosquito Aedes aegypti, el principal vector de los virus que causan el dengue, se encuentra distribuido por todo el territorio nacional, debido a que el $80 \%$ del territorio se encuentra por debajo de los $1800 \mathrm{~m}$ s. n.m., lo que facilita las condiciones para la propagación de este y convierte a Colombia en el segundo país suramericano más afectado por el vector (Santos, Parra-Henao, Silva y Augusto, 2014). Desde la primera aparición de un caso de dengue grave en 1989, se observa la tendencia al incremento en el número de casos; la década de 1990 proporciona la razón de 5,2 casos por 100000 habitantes, mientras que en los últimos años la proporción es de 18,1 casos por cada 100000 habitantes. Según el Instituto Nacional de Salud de Colombia, en el año 2013 se registraron 12882 casos de dengue en el país, aportando a este número 232 casos el departamento de Atlántico y 308 casos el municipio de Barraquilla (Instituto Nacional de Salud, 2013).

Se reconoce la necesidad de realizar un estudio específico en el departamento de Atlántico, Colombia, teniendo en cuenta los datos mencionados anteriormente. Para ello, se escoge el modelo bayesiano por la utilidad que ha tenido para los países latinoamericanos, Brasil y Venezuela, en poblaciones pequeñas, además, el beneficio que proporciona la combinación de los modelos jerárquicos bayesianos con los sistemas de información geográfica, herramienta importante para caracterizar y estimar patrones de enfermedades, considerando simultáneamente estructuras complejas de modelaje jerárquico, factores de riesgo sociodemográfico y factores ambientales (González, Infante y Hernández, 2012). El modelo bayesiano permite el estudio de la enfermedad en el espacio de manera cuantitativa, una alternativa para el análisis de los problemas geográficos, que a diferencia del método clásico o frecuentista cuyo supuesto es que los parámetros del modelo son fijos pero desconocidos, la estadística bayesiana se construye sobre un modelo de probabilidad que enlaza la distribución de los datos a sus parámetros, que son tratados como variables aleatorias que dependen de los supuestos a priori.

Adicionalmente posibilita introducir variables que no son concretamente del sector de salud, pero pueden tener relación con la aparición de casos de dengue para reconocer la influencia de factores específicos sobre las áreas de estudio y los municipios de mayor riesgo, analizar la relación de los casos de dengue con las variables socio-económicas, culturales y físicas del territorio y construir mapas que permiten identificar patrones de ocurrencia y asociación espacial en cada municipio del departamento de estudio. Como objetivo se plantea identificar el mejor modelo para la descripción de los casos de dengue utilizando análisis espaciales totalmente bayesianos, basados en el Criterio de Información de Desviación (DIC), que se calcula a partir de muestras generadas por cadenas de Markov Monte Carlo. Se comparan cuatro modelos para cada año de estudio y se selecciona el de menor DIC.

\section{Descripción del lugar}

El departamento de Atlántico se ubica en el norte de Colombia en la región Caribe, con una superficie 
de $3388 \mathrm{~km}^{2}$ representando el 0,29\% del territorio nacional, la población que se proyectó para el año 2015 fue de 2461001 distribuida en 23 municipios que se encuentran a una altura entre 0 y $219 \mathrm{~m} \mathrm{s.n.m}$. (DANE, 2005). El departamento se encuentra enmarcado dentro de las coordenadas $10^{\circ} 15^{\prime} 36^{\prime \prime}$ y $11^{\circ}$

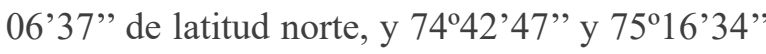
de longitud oeste. Su geografía está representada en un $45 \%$ por terreno montañoso, con serranías y colinas de poca altura, mientras que su hidrografía presenta corrientes mayores, el río Magdalena y el canal del Dique. Adicionalmente, su límite norte cuenta con una extensión aproximada de $90 \mathrm{~km}$ de territorio con el mar Caribe, la demás hidrogra-

\section{FIGURA 1.}

Mapa de zona de estudio, Departamento de Atlántico

Fuente: Elaboración propia, software ArcGIS 10.2 Licencia estudiantil.

E1 95\% de la población es urbana y los municipios que concentran esta población son Barranquilla y Soledad con un $82 \%$, asimismo, el mayor porcentaje de población urbana se concentra a lo largo de los ejes de comunicación del departamento, siendo los municipios de las zonas centro y norte los de mejor conectividad; el municipio de menor conectividad es Piojó, municipio que junto con Luruaco poseen una menor población en su área urbana (Segebre, 2012). La actividad industrial del departamento se concentra en Barranquilla, siendo la industria, la fía la componen arroyos y caños ubicados al sur y occidente del departamento. La región Caribe presenta los períodos más secos en los primeros meses del año, con una precipitación que va de 0 a $250 \mathrm{~mm}$, la cual aumenta para los meses de julio, agosto y septiembre donde alcanza los $1250 \mathrm{~mm}$, a partir de octubre la precipitación varía de 0 a 500 mm (Cadena, Guzmán y Ruíz, 2014). El clima es tropical de tipo estepa y sabana de carácter árido en la desembocadura del río Magdalena y alrededores de su capital Barranquilla, semiárido en las fajas aledañas al litoral y al río Magdalena y semihúmedo desde Sabanalarga hacia el sur. La temperatura media del departamento varía entre $\operatorname{los} 26^{\circ} \mathrm{C}$ y los $28^{\circ} \mathrm{C}$ (Gobernación de Atlántico, 2010).

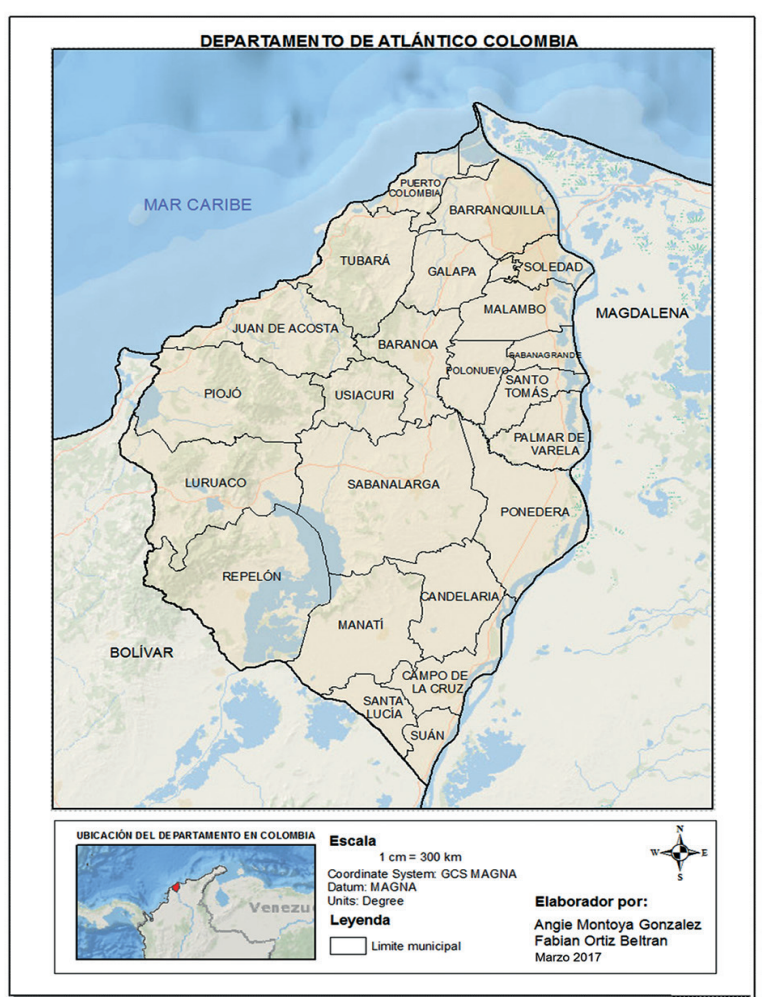

pesca, la ganadería y el comercio las actividades preponderantes. Barranquilla se convierte en el principal puerto de industria del departamento y segundo en 
importancia del país. La cobertura del servicio de agua potable en las cabeceras municipales pasó del $37,7 \%$ en el año 2004 a un $84,1 \%$ al finalizar el año 2006, se divisa un aumento también en la cobertura de educación, pasando de $76 \%$ para el año 2003 a $84 \%$ en el año 2006.

Al ser un importante corredor de movilidad, el territorio facilita el acceso de diferentes grupos armados que se mueven por las riberas del río Magdalena, por sus ciénagas y caños, convirtiendo el departamento un eje de actividades delictivas que promueven el desplazamiento de los habitantes de las zonas rurales a los cascos urbanos, induciendo a una urbanización desordenada en lugares que no abarcan los servicios vitales de vida. El comportamiento del departamento frente al desplazamiento forzado ha sido receptor, debido a las condiciones de puerto y actividad comercial de su capital, los municipios más afectados en el período de 2003 a 2006 fueron Barranquilla, Soledad y Malambo, recibiendo Barranquilla 3166 desplazados, comparados con 273 expulsados de su territorio (ACNUR, s. f.).

\section{Metodología}

Para la construcción del modelo se tuvieron en cuenta los casos registrados de la enfermedad en los municipios del departamento de Atlántico por parte del Sistema Nacional de Vigilancia en Salud Pública SIVIGILA - en los años 2010 a 2013 (SIVIGILA, 2013). El 2010 fue considerado el año de mayor notificación en la historia del dengue en las Américas, con más de 1,6 millones de casos reportados; para Colombia, marcó la más grande epidemia del dengue con más de 150000 casos y 217 muertes confirmadas (Padilla, Rojas y Sáenz Gómez, 2012).

La base de datos se complementó con la proyección de la población para los años de estudio y con datos socioeconómicos aportados por los documentos publicados por la Gobernación de Atlántico, adicionalmente, se generó la variable de tasa de crecimiento en la zona urbana del municipio con base en las proyecciones de población dadas por el DANE. A las variables de estudio antes descritas, se sumó el porcentaje de personas con necesidades básicas insatisfechas (NBI), la cual se obtuvo teniendo en cuenta ciertas características relacionadas con el lugar de vivienda: inadecuadas, en hacinamiento crítico, servicios inadecuados (disponibilidad de agua potable y servicio sanitario para el desecho de excretas), alta dependencia económica (disponibilidad de recursos) y con niños en edad escolar que no asisten a la escuela (Feres y Mancero, 2001). Adicionalmente se incorporan los datos de precipitación y días con lluvia en el año, proporcionados por el Instituto de Hidrología, Meteorología y Estudios Ambientales, Colombia (IDEAM). La descripción de las variables se encuentra en la Tabla 1.

Las variables son organizadas en MS Excel, junto con el archivo en formato shapefile, que contiene la asociación de las variables de base de datos georreferenciadas, estas son cargadas para su procesamiento en el software libre R i386 3.2.2., que permite controlar los parámetros establecidos, incluir código de programación adicional, computar y graficar datos estadísticos, conectado por medio de la librería R2WinBUGS al software estadístico WinBUGS v1.4., utilizado ampliamente en trabajos de epidemiología, enfocado en generar modelos estadísticos espacio temporales, el cual permite realizar inferencia bayesiana por medio de cadenas de Markov Monte Carlo (MCMC). Una cadena Markov es una serie de eventos, donde la probabilidad de que ocurra un evento depende del evento inmediatamente anterior (Del Valle, 2016). 
TABLA 1. DESCRIPCIÓN DE VARIABLES

VARIABLE

Casos de dengue

Población del municipio

Necesidades Básicas

Insatisfechas-NB|

Tasa de crecimiento

Miseria

Hacinamiento

Servicios inadecuados

Dependencia económica

Tasa de escolaridad

Inasistencia

Altura del municipio

Precipitación

Días con lluvia

\section{DESCRIPCIÓN}

Número de personas afectadas por el dengue en los años 2010 a 2013.

Número de personas proyectadas por municipio para los años 2010 a 2013.

Proporción de Personas vs. Hogares, sobre la Población total vs. Total de hogares, que tiene al menos una necesidad básica insatisfecha.

Tasa que indica el crecimiento o decrecimiento de la población que habita en la zona urbana de los municipios del departamento.

Indicador que expresa las características físicas de viviendas consideradas impropias para el alojamiento urbano.

Indicador que capta los niveles críticos de ocupación de los recursos de la vivienda por el grupo que la habita.

Indicador que muestra el no acceso a condiciones vitales y sanitarias mínimas, servicio de acueducto y alcantarillado.

Indicador indirecto sobre los niveles ingreso de las viviendas.

Porcentaje de la cobertura bruta de educación para el municipio.

Indicador que mide la satisfacción de necesidades educativas mínimas para la población infantil.

Altura media sobre el nivel del mar para cada municipio del departamento del Atlántico.

Milímetros de agua que caen a la superficie terrestre desde la atmósfera.

Número de lluvias en el periodo de un año.

Fuente: Elaboración propia.

Se consideró una cadena de Markov de Monte Carlo, bajo métodos computacionales, de la cual se realizaron 30000 iteraciones.

\subsection{Cálculo de la tasa de mortalidad estandarizada (SMR)}

Con la base de datos organizada, se halló la tasa de mortalidad estandarizada (SMR) como una alterativa de aislar el ruido presente de la variable endógena

Casos de dengue, ajustándola a la población de donde se presentan los casos (Waller y Gotway, 2004). La SMR es un estadístico muy difundido para representar los patrones espaciales de la distribución de una enfermedad, se expresa como la proporción entre el número de casos observados y el número de casos esperados (Pfeiffer, Robinson, Stevenson,
Stevens, Rogers y Clements, 2008); para su cálculo, el trabajo toma el número de casos de dengue y el número total de habitantes por municipio.

$$
S M R_{i}=\frac{O_{i}}{e_{i}}
$$

\section{Fórmula 1. Cálculo de la SMR}

Donde $O_{i}$ es el número de casos de dengue presentados en el municipio $i$ y el número de casos esperados de dengue, se define como:

$$
e_{i}=n_{i}
$$

Fórmula 2. Cálculo de número de esperados de dengue

Donde $n_{i}$ es el número de total de habitantes del municipio $i$. 


\subsection{Análisis exploratorio de las variables}

El análisis exploratorio de las variables permite determinar el comportamiento individual de las variables y su relación con la variable endógena. Identificar la mejor distribución para la variable endógena depende de una buena elección de la matriz de pesos espaciales, para esto se aplican las pruebas de autocorrelación espacial Contraste de I de Moran Global, Contraste de I de Moran Global Simulada, Contraste de I de Moran Global adaptada al enfoque Empírico Bayesiano, y un Contraste de C de Geary Global (Murcia y Sarmiento, 2015)

Para la descripción del comportamiento de las variables se generaron gráficos descriptivos globales como los mapas de caja, cuya interpretación permite identificar datos atípicos y patrones geográficos, posteriormente se produjeron los mapas de autocorrelación espacial local (LISA), que permiten identificar agregaciones espaciales. Los mapas fueron generados el software GeoDa enfocado en el análisis espacial.

\subsection{Modelo bayesiano}

El modelo bayesiano permite la toma lógica de decisiones bajo incertidumbre, posibilita incorporar hipótesis científicas en el análisis por medio de las distribuciones a priori, que se aplican en problemas de estructura compleja y pueden ser difíciles de manejar por medio de métodos convencionales (Pfeiffer et al., 2008).

Aplicando el teorema de Bayes, la distribución a posteriori es proporcional al producto entre la función de probabilidad (distribución de probabilidad condicional de los datos observados dado el vector de parámetros ), y la distribución a priori de los parámetros, que es definida por el investigador sin usar los datos. Así se tiene la expresión:

$$
f \theta|0 \alpha f 0 \quad \theta f \theta ; c=f 0| \theta
$$

Fórmula 3. Inferencia bayesiana

Donde $c$ es una constante de proporcionalidad, cuya función es asegurar que la distribución a posteriori integre al valor de 1. Las distribuciones a priori y de probabilidad proveen dos fuentes de información sobre cualquier problema. La función de probabilidad $f O$ $\theta$, da información sobre el parámetro a través de los datos, mientras que la distribución a priori $f \theta$ lo hace a través de creencias o suposiciones previas. Cuando hay gran cantidad de información, la probabilidad contribuirá más en la estimación del riesgo relativo. Cuando se cuenta con pocos datos la distribución a priori dominará el análisis (Lawson, 2008).

De acuerdo con el modelo jerárquico bayesiano y con el ánimo de incluir los efectos aleatorios y el componente espacial se puede reescribir:

$$
f \theta_{\psi} \psi, \beta|0 \quad \alpha f 0| \beta, \psi f \psi \theta_{\psi} f \beta f \theta_{\psi}
$$

Fórmula 4. Fórmu la del modelo lineal generalizado.

Donde $\beta$ es un vector de efectos fijos relacionados con las covariables que explican la variable respuesta $0, \psi$ corresponde al vector de efectos aleatorios y $\theta_{\psi}$ al vector de parámetros que definen la correlación espacial.

El análisis bayesiano asume que todos los parámetros del modelo se comportan como variables aleatorias, no obstante, en el contexto de los modelos mixtos, los efectos fijos $(\beta)$ corresponden a parámetros invariables, por tanto, el comportamiento de las covariables es el mismo para toda la zona objeto de estudio. Los efectos aleatorios, en cambio, son parámetros que cambian entre las regiones, ya que 
dependen de las distribuciones de los datos observados para cada población (Waller y Gotway, 2004).

El enfoque bayesiano contempla los efectos fijos denominados por los betas que acompañan a las variables del modelo. Se evaluaron 4 modelos de los cuales se analizó el criterio de información de desviación (DIC) y, sobre la base de esa información, se seleccionó el modelo con la DIC más baja como el modelo que mejor estima el riesgo de dengue para cada año de estudio.

\section{Resultados}

Selección de la mejor matriz de pesos espaciales, según los criterios de contigüidad: Torre, Reina,
Triangulación de Delaunay (TDD), Esfera de influencia (EDI), Gráfica de Gabriel (GDG), Vecinos Relativos (VVRR), y el criterio de los n-vecinos más cercanos, $\mathrm{n}=1-K N N 1, \mathrm{n}=2-K N N 2, \mathrm{n}=3-K N N 3$ y $\mathrm{n}=4-K N N 4$. (Tabla 2).

Se selecciona la matriz de pesos con el menor valor de índice AIC en cada año, pues esta es la que mejor representa la dependencia espacial presente en la variable endógena. Con la matriz de KNN1, se evalúan las pruebas de autocorrelación global para determinar si en promedio hay evidencia de correlación espacial en el área de estudio. (Tabla 3).

El p-valor de cada una de las pruebas indica que no hay suficiente evidencia estadística para decir que no

TABLA 2. CRITERIOS DE CONTIGÜIDAD

\begin{tabular}{cccccccccccc}
$\begin{array}{c}\text { CRITERIO/ } \\
\text { AÑO }\end{array}$ & TORRE & REINA & TDD & EDI & GDG & VVRR & KNN1 & KNN2 & KNN3 & KNN4 \\
\hline 2010 & 14,73 & 18,40 & 16,67 & 21,68 & 25,99 & 18,03 & 10,86 & 21,91 & 18,06 & 12,12 \\
2011 & $-4,93$ & $-4,01$ & 1,88 & 0,004 & $-0,99$ & $-9,66$ & $-18,03$ & $-8,26$ & $-11,17$ & $-3,82$ \\
2012 & $-0,17$ & $-1,74$ & $-10,22$ & $-6,00$ & $-8,34$ & 1,63 & $-48,31$ & 2,45 & $-12,80$ & $-9,91$ \\
2013 & $-44,46$ & $-33,43$ & $-36,22$ & $-42,93$ & $-36,55$ & $-38,82$ & $-58,22$ & $-41,20$ & $-37,88$ & $-38,70$ \\
\hline
\end{tabular}

TABLA 3. PRUEBAS DE AUTOCORRELACIÓN GLOBAL

\begin{tabular}{ccccccccc}
\multirow{2}{*}{ AÑO } & \multicolumn{2}{c}{ I DE MORAN } & \multicolumn{2}{c}{$\begin{array}{c}\text { I DE MORAN } \\
\text { SIMULADA }\end{array}$} & \multicolumn{2}{c}{$\begin{array}{c}\text { I DE MORAN } \\
\text { ADAPTADA A EB }\end{array}$} & \multicolumn{2}{c}{ C DE GEARY } \\
\cline { 2 - 9 } & Estadístico & p-valor & Estadístico & p-valor & Estadístico & p-valor & Estadístico & p-valor \\
\hline 2010 & 0,10636395 & 0,5349 & 0,1063639 & 0,586 & 0,56279 & 0,130 & 0,95806294 & 0,8814 \\
2011 & 0,07490760 & 0,5829 & 0,0749076 & 0,685 & 0,035293 & 0,360 & 0,6369582 & 0,2357 \\
2012 & 0,52459467 & 0,0027 & 0,5245947 & 0,031 & 0,57362 & 0,004 & 0,4685397 & 0,1060 \\
2013 & 0,10797122 & 0,5410 & 0,1079712 & 0,577 & 0,097616 & 0,316 & 0,62755895 & 0,1741 \\
\hline
\end{tabular}

Fuente: Elaboración propia. 


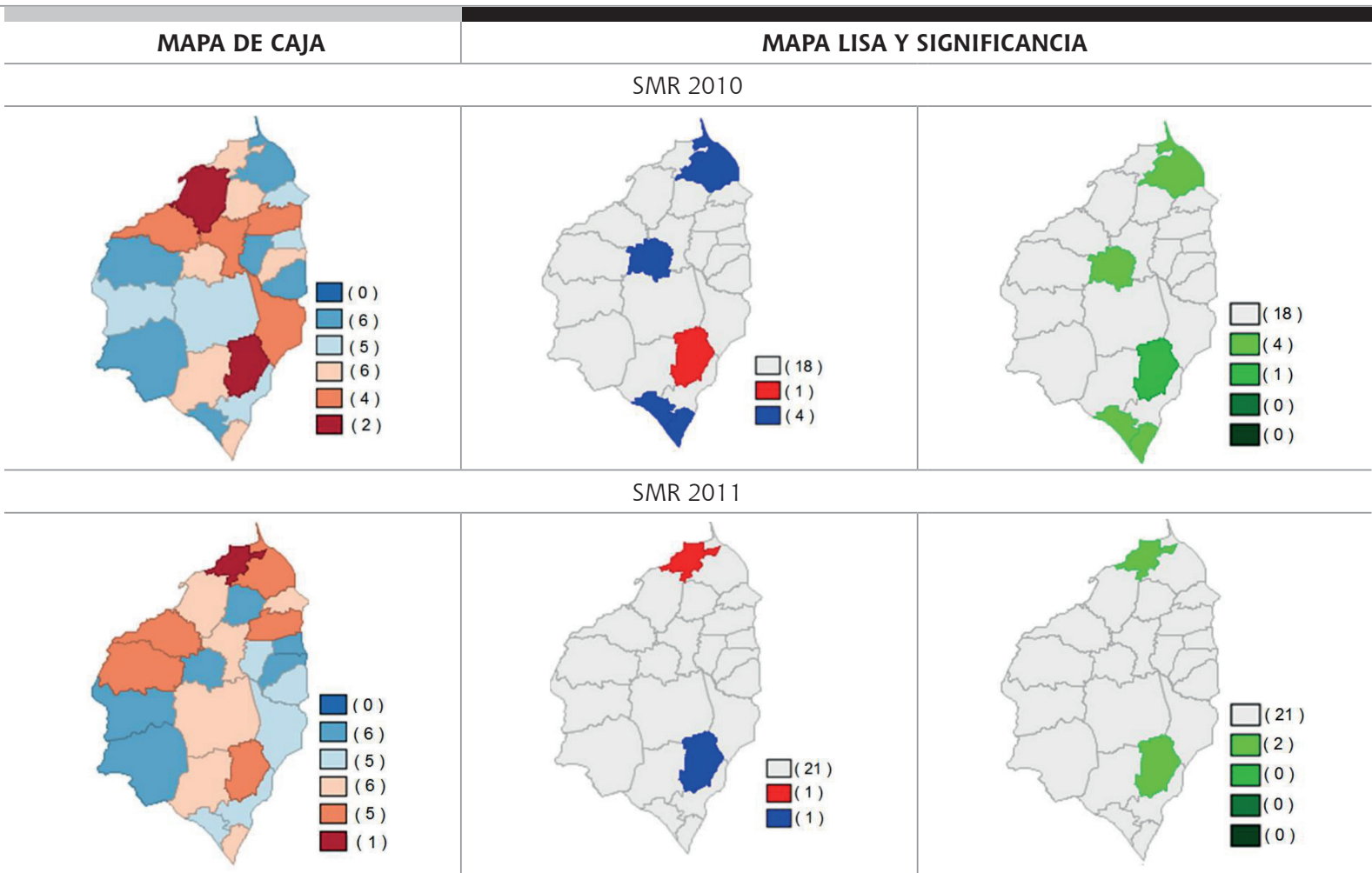

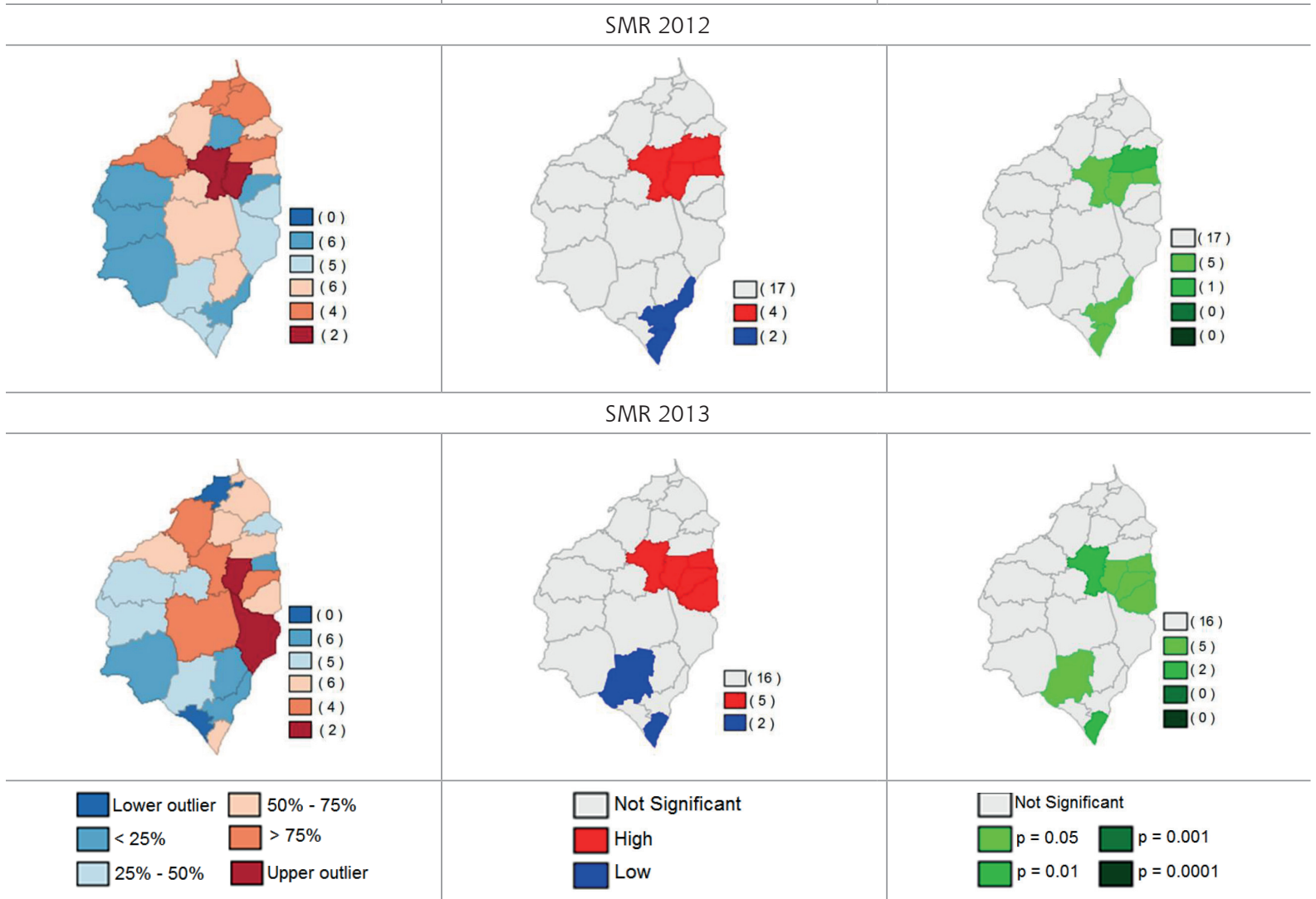

Fuente: Elaboración propia. 
hay estructura espacial en los casos de dengue en el departamento de Atlántico, sin embargo, los coeficientes de autocorrelación espacial fueron positivos para cada uno de los años, lo que sugiere que los municipios que presentan valores altos de la SMR, es decir, con mayor número de casos de dengue según población municipal, se encuentran rodeados por municipios con valores altos y por consiguiente, aquellos con valores bajos de la SMR, es decir, con menor número de casos de dengue según población municipal, se encuentran rodeados por municipios con valores bajos.

En el año 2010, la base de datos de SIVIGILA reportó 716 casos de dengue en el departamento de Atlántico, donde los municipios que más presentaron casos de dengue fueron Barranquilla con 113, Malambo con 119 y Soledad con 219 casos, sin embargo, en el mapa de caja para el 2010, mostró dos municipios con valores atípicos altos para la SMR, Tubará y Candelaria. Tubará contó con 22 casos reportados con una población de 10980 habitantes, es decir, una razón aproximada de 1 persona afectada por dengue por cada 500 habitantes, a diferencia de Barranquilla que aparece en el menor cuartil $(<25 \%)$, con una razón de aproximadamente 1 personada afectada por cada 10500 habitantes.

Los casos de dengue reportados en el 2011 fueron 773 , los municipios que reportaron más casos fueron Barranquilla y Soledad con 375 y 166 casos de dengue respectivamente. Barranquilla se ubica en el cuartil más alto (> 75\%), mientras que Soledad se sitúa en el tercer cuartil (50\%-75\%), sin embargo, aparece un municipio con un alto valor de la SMR como un dato atípico, Puerto Colombia, municipio que reportó 44 casos de dengue, con una razón de 1 persona afectada por dengue por cada 125 habitantes.

Los municipios Baranoa y Polonuevo se situaron en los valores más altos atípicos de la SMR en el año 2012. Según las proyecciones de población del DANE, en el año 2012 la población de Baranoa fue de 56037 habitantes, de los cuales se reportaron 55 afectados por dengue, mientras que Polonuevo reportó 36 afectados por dengue en una población 14889 habitantes. En el 2012, el municipio con más casos de dengue fue Barranquilla con 593 afectados, situándolo en el cuarto cuartil (> 75\%).

El año con el reporte más alto de casos de dengue en el departamento de Atlántico fue el 2013, con una cifra de 5217 afectados. Polonuevo con 69 casos y Ponedera con 96 casos fueron los municipios con la SMR más alta, el municipio de Polonuevo con una razón de 1 habitante afectado por dengue por cada 218 habitantes y el municipio de Ponedera con una razón de 1 habitante afectado por dengue por cada 225 habitantes. Barranquilla y Soledad, municipios que aportaron más de la mitad de los casos de dengue, se situaron en el tercer y segundo cuartil, respectivamente.

Los mapas de caja no mostraron comportamientos espaciales claros de manera global. Un municipio con valores altos de la SMR puede ubicarse en el norte o sur del departamento, así mismo los municipios con valores bajos de la SMR se presentan distribuidos por toda el área de la zona de estudio sin ningún patrón especial. Los mapas de autocorrelación espacial (LISA) y de significancia no muestran agregaciones espaciales para los dos primeros años de estudio, sin embargo, para el 2012 y 2013, los municipios con valores altos de la SMR se encuentran rodeados por valores altos y los municipios con valores bajos de la SMR están rodeados por municipios con valores bajos. (Tabla 5).

La variable Tasa de crecimiento muestra el porcentaje de crecimiento del casco urbano respecto al año anterior. En el mapa de caja no se evidencia 


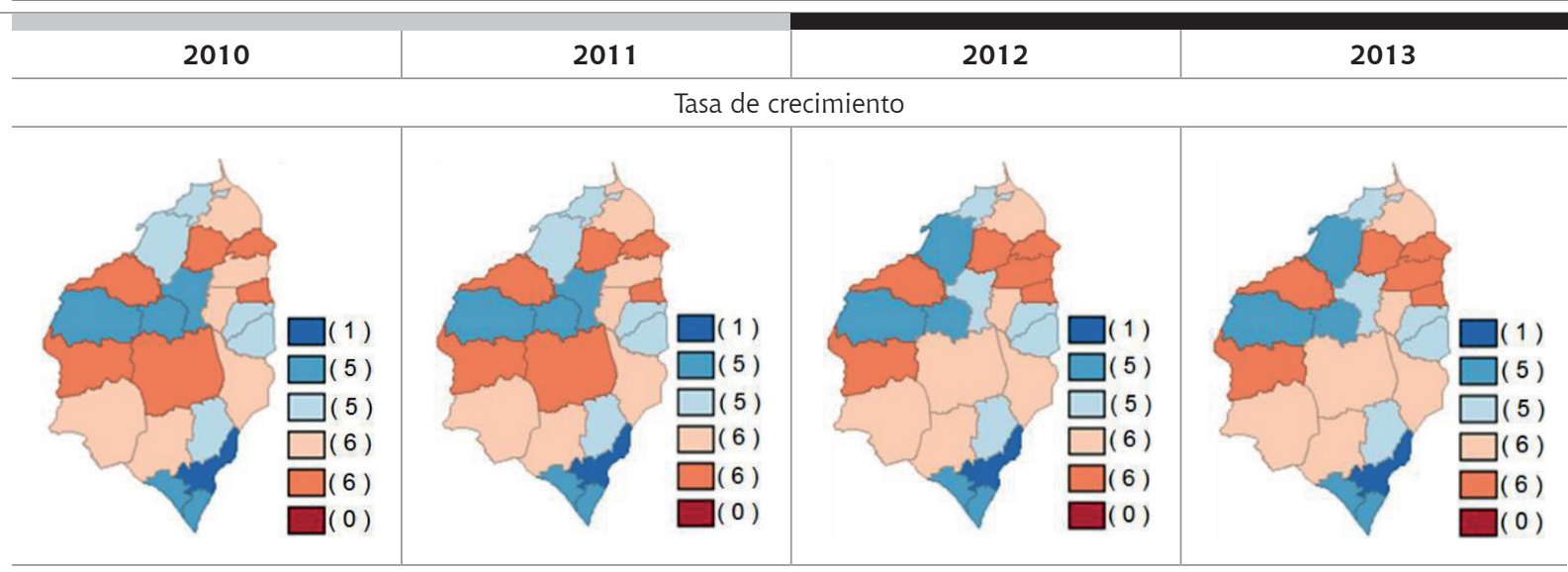

Miseria

Fuente: Elaboración propia.

una asociación espacial de los municipios, sin embargo, los municipios Galapa, Soledad, Malambo, Sabanagrande, Luruaco y Juan de Acosta, que se encuentran en el cuartil más alto ( $>75 \%)$, están ubicados en la zona centro-norte del departamento. Esta variable se espera sea de influencia en el modelo debido a que el crecimiento del casco urbano influye en la cobertura de servicios públicos y por ende en el posible almacenamiento de agua en tanques.

Las variables Miseria, Servicios inadecuados y Dependencia económica concentran sus valores 
más altos en los municipios de la zona sur del departamento, siendo Repelón, Manatí, Santa Lucía, Candelaria y Campo de la Cruz los municipios que muestran mayor porcentaje de viviendas inadecuadas, no obstante, poca cobertura de los servicios de agua potable y de alcantarillado y escasos ingresos económicos. La Inasistencia escolar ubica a pocos municipios en el cuarto cuartil, sin embargo, son tres municipios ubicados en los valores atípicos altos, Ponedera, Campo del Cruz y Sabanagrande, los que indican un alto porcentaje de población infantil vulnerable. La variable Hacinamiento permite notar que los municipios en la zona norte no presentan porcentajes altos en hacinamiento crítico, una buena relación entre el número de personas que habitan y el número de dormitorios por cada vivienda. Los municipios Manatí, Candelaria, Campo de la Cruz, Santa Lucía y Suan son los que tienen un porcentaje más alto de NBI, adicionalmente se encuentran ubicados al sur del departamento, por otro lado, los municipios de Puerto Colombia, Barranquilla, Soledad y Tubará presentan porcentajes bajos en esta variable y además se ubican al norte del departamento.

Los municipios con mayor Precipitación y Días con lluvia se ubican en la zona centro del departamento, y corresponden a Piojó, Malambo y Sabanagrande, los cuales presentan una precipitación entre $1184,7 \mathrm{~mm}-$ 1277,3 mm y aproximadamente 93 días de lluvia al año.
Con el enfoque bayesiano, se asume que los parámetros se comportan como variables aleatorias, en este caso, se contemplan los efectos fijos como parámetros invariables y los efectos aleatorios son cambiantes entre región, ya que depende de los valores observados en estas (Murcia y Sarmiento, 2015). Se estima el modelo jerárquico bayesiano con el uso del software WinBUGS, el cual proporciona el criterio de información de desviación (DIC) que permite comparar modelos bayesianos del mismo año.

Se generaron cuatro modelos para cada año, el mejor modelo para el estudio de la enfermedad del dengue fue el modelo que presentó el valor más bajo del DIC. Los modelos fueron: (1) el primer modelo contempló todas las variables de estudio, las variables sociales, económicas y geográficas; (2) el segundo modelo se centra en la búsqueda de la incidencia de las variables geográficas en los casos de dengue; (3) el tercer modelo agrupa las variables que definen la calidad de vida de las personas según el estado y servicios disponibles de las viviendas, ingresos económicos y nivel de educación; y (4) el cuarto modelo contempla las variables relacionadas con el crecimiento desorganizado del casco urbano y por consiguiente poca disponibilidad de recursos y falta de acceso a los servicios públicos.

\section{TABLA 6. MODELOS JERÁRQUICOS BAYESIANOS AÑOS 2010-2013}

\begin{tabular}{ccccc}
\multicolumn{5}{c}{ DIC } \\
\hline Modelo & 2010 & 2011 & 2012 & 2013 \\
Modelo 1 & 78895 & 86787 & 46378 & 79074 \\
Modelo 2 & 124219 & 86128 & 84490 & 126347 \\
Modelo 3 & 109352 & -366389 & 16900 & 113371 \\
Modelo 4 & -2342 & 114586 & 44009 & -109903 \\
\hline
\end{tabular}

Fuente: Elaboración propia. 
Modelo (1) con todas las variables de estudio: beta1-NBI, beta2-Días con lluvia, beta3-Precipitación, beta4-Altura del municipio, beta5-Tasa de escolaridad, beta6-Miseria, beta7-Servicios, beta8-Inasistencia escolar, beta9-Dependencia económica, beta10-Tasa de crecimiento, beta11-Hacinamiento.

Modelo (2) variables físicas: beta1-Días con lluvia, beta2-Precipitación, beta3-Alrura del municipio (Mena et al., 2011).

Modelo (3) variables socio-económicas: beta1-Miseria, beta2- Servicios inadecuados, beta3- Inasistencia escolar, beta4-Dependencia económica, beta-5- Hacinamiento (Honorato et al., 2014)

Modelo (4) variables producto de la urbanización no planificada: beta1-Servicios inadecuados, beta2-Dependencia económica, beta3-Tasa de crecimiento (Silveira y de Barros, 2015).

Para los años 2010 y 2013, el modelo que mejor explicó los casos presentados de dengue fue el (4), siendo el crecimiento del casco urbano una razón que puede influir en la poca cobertura de servicios públicos y, así, el posible almacenamiento de agua en tanques se convierte en el factor que incidió en la aparición de casos de dengue en el departamento. Adicionalmente, para estos dos años, el segundo modelo que mejor explica los casos de dengue es el (1), donde se incorporan todas las variables.

El modelo (3) fue el que mejor explicó los casos presentados de dengue en los años 2011 y 2012, siendo las condiciones de vivienda, la calidad de vida, los recursos económicos y el acceso a educación de los habitantes los factores relevantes en la incidencia del dengue. $\log \left(0_{i} \theta s_{i}=2.163-0.008296\right.$ ServiciosInadecuados - 0.05876DependenciaEconómica + 0.003416 TasaCrecimiento $+0.1576+2.355$ )

Fórmula 5. Fórmula mejor modelo año 2010.

$\log \left(0_{i} \theta s_{i}=-1.863-0.8033\right.$ Miseria +0.2835 ServiciosInadecuados +0.4743 Inasistencia + 0.04712 DependenciaEconómica -0.4956 Hacinamiento $+1.018+6.83)$

Fórmula 6. Fórmula mejor modelo año 2011.

$$
\log \left(0_{i} \theta s_{i}=2.392-0.008462\right. \text { Miseria - }
$$
0.0675 ServiciosInadecuados +0.05638 Inasistencia

+ 0.1099DependenciaEconómica -0.3207

Hacinamiento $+1.018+1.859)$

Fórmula 7. Fórmula mejor modelo año 2012.

$\log \left(0_{i} \theta s_{i}=-2.631-0.02493\right.$ ServiciosInadecuados +0.1166 DependenciaEconómica +0.7825 TasaCrecimiento $+0.03463+0.568)$

Fórmula 8. Fórmula mejor modelo año 2013.

En la Tabla 7 se presentan los mapas de probabilidad basados en el enfoque Choynowski. La tonalidad más oscura muestra a los municipios con probabilidad de presentar números altos o bajos en los casos de dengue. Las probabilidades de tener número altos en los casos de dengue tienen una relación con el rango latitudinal. En el año 2010, Tubará, Galapa Juan de Acosta, Baranoa y Soledad, presentan la probabilidad más alta. En el 2011, el municipio con mayor probabilidad en tener número altos es Malambo, el cual se encuentra geográficamente debajo de Soledad. Para el año 2012, el municipio de Malambo junto con Baranoa y Polonuevo, dos municipios con latitud más bajas que este, son los que tienen mayor probabilidad de tener un número altos de 


\section{TABLA 7. MAPA DE PROBABILIDAD DE CHOYNOWSKI PARA LA VARIABLE SMR}

(1)

Fuente: Elaboración propia.

casos de dengue. Para finalizar, en el año 2013, los municipios con mayor probabilidad de presentar número altos son Polonuevo, que ya se había presentado en el 2012, junto con Sabanalarga y Ponedera, los cuales tienen una menor latitud.

\section{Discusión}

La inclusión de los años 2010-2013 en el presente trabajo permitió ver que la enfermedad no tiene un comportamiento de fácil descripción, los municipios más afectados por el dengue no fueron descritos por un patrón espacial en cada año, además, las variables que mejor describieron el modelo de riesgo de la enfermedad no fueron las mismas para los años de estudio, dificultando realizar hipótesis para los años siguientes. Escoger cuatro años de estudio conse- cutivos no es una muestra lo suficiente buena para identificar patrones temporales de la enfermedad, a diferencia de los modelos temporales para la enfermedad del dengue aplicados en la ciudad de Can Tho en Vietnam en el periodo de 2003-2010, que dan la oportunidad de identificar periodos de tiempo de mayor riesgo y así lograr establecer predicciones acertadas de la enfermedad (Phung et al., 2015).

Se encontró una relación significativa sobre las variables de servicios inadecuados y dependencia económica para dos de los años de estudio, variables que fueron también relevantes en el modelo bayesiano para los casos de dengue en Espírito Santo Brasil (Honorato et al., 2014), donde el modelo con menor DIC fue el que incorporó el efecto espacial con las variables de servicio de alcantarillado e ingresos económicos. 
En Colombia, el desplazamiento de la población provoca el incremento de las áreas urbanas donde no se garantiza calidad de vida por la falta de cobertura de servicios públicos y equipamientos, lo que puede promover la práctica de almacenamiento de agua en tanques (Espinosa, 1998). La Secretaría Departamental de Salud del Atlántico asegura que las costumbres culturales como la reserva de agua en albercas u otros medios son un aspecto de difícil control, el tamaño de estas obstaculiza el lavado periódico y necesario para evitar el desarrollo de las larvas y posteriormente la propagación del mosquito adulto (Mosquera, Obregón, Lloyd, Orozco y Peña, 2010). Un modelo del estilo Takagi-Sugeno compuesto por ecuaciones diferenciales parciales, bajo simulaciones en un periodo de verano, evidencia que el aumento de envases de reproducción de mosquitos, producto de las costumbres culturales de la población o de la lluvia que se repose en el territorio, es la razón del aumento de criaderos de mosquitos, y por ende el aumento del riesgo de dengue (Silveira y de Barros, 2015). Incorporar en el presente trabajo un modelo que contempló el crecimiento del casco urbano y la evidencia de posibles tanques de agua que aumenten la reproducción del mosquito permitió ver la influencia de este fenómeno en el aumento del riesgo de la enfermedad del dengue en el departamento de Atlántico para los años 2010 y 2013.

El estudio de los factores asociados al dengue en Costa Rica, por medio de regresiones de Poisson, revela que la altitud, la temperatura y el índice de pobreza humana, tienen relación con la incidencia del dengue (Mena, Aroyo, Bonilla-Carrión y Calderón-Arguedas, 2011). Sin embargo, la extensión geográfica del departamento de Atlántico no permitía encontrar mayores cambios en la altura y temperatura entre sus municipios, por lo que fueron variables que no fueron relevantes en el modelado del riesgo del dengue en ninguno de los años de estudio.

Respecto al modelado totalmente bayesiano de la enfermedad del dengue, se resalta el uso de la SMR y su relación con las demás variables, el uso de esta aleja el ruido que produce el reporte de grandes cifras de casos de dengue en poblaciones grandes. Los municipios del departamento de Atlántico, Barranquilla con 3944 casos de dengue y Soledad con 1594 casos dengue, reportados en los 4 años de estudio, los convierten en los municipios más afectados por la enfermedad a simple vista. Sin embargo, la metodología propuesta por Waller y Gotway (2004) de hallar SMR permitió ajustar los casos de dengue al total de habitantes por municipio y así observar las poblaciones más afectas por la enfermedad, que fueron los municipios Tubará, Candelaria, Puerto Colombia, Baranoa, Polonuevo y Ponedera.

Las políticas de control del virus no han tenido el alcance deseado en los países latinoamericanos, convirtiendo a Colombia, Venezuela y México en países con gran número de casos de dengue (Monsalve et al., 2010). Los programas de atención apuntan a controlar las prácticas culturales de almacenamiento de agua en diferentes recipientes, la cual es una labor importante pero no suficiente para el control de la enfermedad. Es importante generar campañas en contra de la desigualdad socio-económica, mejorando los niveles de calidad de vida, así como acceso a educación que promueva el aumento de personas con carreras profesionales que apunten a mejores empleos.

\section{Conclusiones}

Se logró identificar el mejor modelo bayesiano para cada uno de los años de estudio, en los cuales se 
identificaron las variables que más influyen en los casos de dengue en el departamento de Atlántico, es decir, los niveles sociales de la población, las viviendas inadecuadas, la falta de servicios de acueducto y alcantarillado, la población infantil que no asiste al colegio y el deficiente ingreso económico de los hogares. Adicionalmente, las personas que se desplazan a las áreas urbanas donde no hay una planeación del territorio se ubican en lugares sin acceso a los servicios básicos y por ende adquieren prácticas desfavorables que promueven la reproducción del mosquito. Hay municipios donde se debe priorizar la atención. Si bien es cierto que Barranquilla y Soledad son los municipios que más casos de dengue reportan y alarman a las autoridades, el departamento de Atlántico tiene municipios donde la razón de personas afectadas por dengue es mayor; aunque las cifras de casos reportados no parezcan impresionantes son las áreas de mayor riesgo de la enfermedad. Las campañas y programas de atención para prevenir la enfermedad del dengue deben apuntar a mejorar la estructura social del departamento, que genera desigualdades en este nivel así como en el económico. 


\section{Referencias}

Aburas, H. M., Cetiner, B. G. y Sari, M. (2010). Dengue confirmed-cases prediction: A neural network model. Expert Systems with Applications, 37(6), 4256-4260. Recuperado de http://doi.org/10.1016/j.eswa.2009.11.077

Alto Comisionado de las Naciones Unidas para los Refugiados (ACNUR). (s. f.). Diagnóstico departamental Atlántico. Recuperado de http://www.acnur.org/t3/ uploads/media/COI_2163.pdf?view $=1$

Bhatt, S., Gething, P. W., Brady, O. J., Messina, J. P., Farlow, A. W., Moyes, C. L., ... Hay, S. I. (2013). The global distribution and burden of dengue. Nature, 496(7446), 504-507. Recuperado de http://doi.org/10.1038/nature12060

Brady, O. J., Gething, P. W., Bhatt, S., Messina, J. P., Brownstein, J. S., Hoen, A. G., ... Hay, S. I. (2012). Refining the Global Spatial Limits of Dengue Virus Transmission by Evidence-Based Consensus. PLoS Neglected Tropical Diseases, 6(8), e1760. Recuperado de http://doi.org/10.1371/journal.pntd.0001760

Cadena, M., Guzmán, D. y Ruíz, J. F. (2014). Regionalización de Colombia según la estacionalidad de la precipitación media mensual, a través de análisis de componentes principales (ACP). Grupo de modelamiento de tiempo, clima, y escenarios de cambio climático. Subdirección de meteorología-IDEAM. Recuperado de http://www.ideam.gov.co/documents/

Departamento Administrativo Nacional de Estadística (DANE). (2005). Recuperado de http://www.dane.gov.co/index.php/poblacion-y-demografia/proyecciones-depoblacion

Del Valle, J. A. (2016). Introducción a las Cadenas o Procesos de Markov. Recuperado de http://www.ingenieria.unam.mx/javica1/ingsistemas2/Simulacion/Cadenas_de_ Markov.htm

Dom, N. C., Hassan, A. A., Latif, Z. A. \& Ismail, R. (2013). Generating temporal model using climate variables for the prediction of dengue cases in Subang Jaya, Malaysia. Asian Pacific Journal of Tropical Disease, 3(5), 352-361. Recuperado de http://doi.org/10.1016/S2222-1808(13)60084-5

Echavarria, A. y Quintero, O. L. (2012). Estudio de los factores climaticos y geográficos que influyen en la presencia de casos de dengue y criaderos de Aedes aegypti en el municipio de Bello. Recuperado de http://repository.eafit.edu.co:80/ handle/10784/4614

Espinosa, R. (1998). La problemática de los desplazados en el Atlántico. Recuperado de http://www.eltiempo.com/archivo/documento/MAM-824868

Feres, J. C. y Mancero, X. (2001). El método de las necesidades básicas insatisfechas (NBI) y sus aplicaciones en América Latina. CEPAL. Recuperado de http://dds.cepal.org/ infancia/guide-to-estimating-child-poverty/bibliografia/capitulo-III/Feres\%20 Juan\%20Carlos\%20y\%20Xavier\%20Mancero\%20\%282001b\%29\%20El\%20 metodo\%20de\%20las\%20necesidades\%20basicas\%20insatisfechas\%20 \%28NBI\%29\%20y\%20sus\%20aplicaciones\%20en\%20America\%20Latina.pdf 
Gobernación de Atlántico. Secretaría de planeación Atlántico (2010). Anuario estadístico del Atlántico 2010. Recuperado de http://atlantico.gov.co

González, R., Infante, S. y Hernández, A. (2012). Modelos jerárquicos espacio temporales para mapear riesgos relativos de dengue, en el Municipio Girardot, Estado Aragua, Venezuela. Boletín de Malariología y Salud Ambiental, 52(1), 33-45. Recuperado de http://www.scielo.org.ve/scielo.php?script =sci arttext\&pid =S1690-46482012000100004\&lng =es\&nrm = iso\&tlng =es

Honorato, T., Lapa, P. P. de A., Sales, C. M. M., Reis-Santos, B., Tristão-Sá, R., Bertolde, A. I. y Maciel, E. L. N. (2014). Spatial analysis of distribution of dengue cases in Espírito Santo, Brazil, in 2010: use of Bayesian model. Revista Brasileira de Epidemiologia, 17, 150-159. Recuperado de http://doi.org/10.1590/18094503201400060013

Instituto Nacional de Salud. (2013). Situación del dengue en Colombia, 2013. Recuperado de http://www.ins.gov.co/noticias/paginas/situaci\%C3\%B3n-deldengue-en-colombia-hasta-el-16-de-febrero-de-2013.aspx\#.V2n3vVnKuU4

Lawson, A. (2008). Bayesian Disease Mapping: Hierarchical Modeling in Spatial Epidemiology. Boca Raton: Chapman and Hall/CRC.

Mena, N., Troyo, A., Bonilla-Carrión, R. y Calderón-Arguedas, Ó. (2011). Factors associated with incidence of dengue in Costa Rica. Revista Panamericana de Salud Pública, 29(4), 234-242. http://doi.org/10.1590/S1020-49892011000400004

Ministerio de Educación Nacional. (2012) Región Caribe (Costa Atlántica) en Educación. Recuperado de http://www.mineducacion.gov.co/1621/ articles-283230_archivo_pdf_perfil.pdf

Monsalve, N. C., Rubio-Palis, Y. y Pérez, M. E. (2010). Modelaje bayesiano espaciotemporal de factores asociados con la incidencia del dengue en el área metropolitana de Maracay, Venezuela. Boletín de Malariología y Salud Ambiental, 50(2), 219-232. Recuperado de http://www.scielo.org.ve/scielo.php?script=sci_ arttext\&pid $=\$ 1690-46482010000200006 \& \operatorname{lng}=p t \& n r m=$ iso\&tlng $=$ es

Mosquera, M., Obregón, R., Lloyd, L. S., Orozco, M. y Peña, A. (2010). Reflexiones sobre el alcance de la investigación formativa en comunicación en salud en los programas de prevención y control de dengue. El caso de Barranquilla (Colombia). Investigación y Desarrollo, 18(1), 186-217. Recuperado de https:// dialnet.unirioja.es/servlet/articulo? codigo $=3685496$

Murcia, C. E. y Sarmiento F. J. (2015). Modelos bayesianos para describir el comportamiento del cáncer gástrico en Colombia en el periodo 2005-2012 (tesis de pregrado). Universidad Distrital Francisco José de Caldas, Bogotá, Colombia.

Organización Mundial de la Salud (OMS). (2012). Dengue. Recuperado de http://www. who.int/topics/dengue/es/

Padilla, J. C., Rojas, D. P. y Sáenz Gómez, R. (2012). Dengue en Colombia: epidemiología de la reemergencia a la hiperendemia. Bogotá, Colombia: Guías de Impresión Ltda.

Pfeiffer, D., Robinson, T., Stevenson, M., Stevens, K., Rogers, D. y Clements, A. (2008) Spatial Analysis in Epidemiology. Oxford: University Press 
Phung, D., Huang, C., Rutherford, S., Chu, C., Wang, X., Nguyen, M., ... Manh, C. D. (2015). Identification of the prediction model for dengue incidence in Can Tho city, a Mekong Delta area in Vietnam. Acta Tropica, 141, Part A, 88-96. http://doi. org/10.1016/j.actatropica.2014.10.005

Santos, S. L. dos, Parra-Henao, G., Silva, M. B. C. \& Augusto, L. G. da S. (2014). Dengue in Brazil and Colombia: a study of knowledge, attitudes, and practices. Revista Da Sociedade Brasileira de Medicina Tropical, 47(6), 783-787. http://doi. org/10.1590/0037-8682-0048-2014

Segebre, J. A. (2012). Plan de desarrollo 2012-2015. Gobernación de AtlánticoAtlántico más social. Recuperado de http://www.atlantico.gov.co/images/stories/ plan_desarrollo/plan_desarrollo_2012-2015.pdf

Silveira, G. P. y de Barros, L. C. (2015). Analysis of the dengue risk by means of a Takagi-Sugeno-style model. Fuzzy Sets and Systems, 277, 122-137. https://doi. org/10.1016/j.fss.2015.03.003

SIVIGILA. (2013). Vigilancia Rutinaria. Recuperado de http://www.ins.gov.co/lineas-deaccion/Subdireccion-Vigilancia/sivigila/Paginas/vigilancia-rutinaria.aspx

Torres, C., Barguil, S., Melgarejo, M. y Olarte, A. (2014). Fuzzy model identification of dengue epidemic in Colombia based on multiresolution analysis. Artificial Intelligence in Medicine, 60(1), 41-51. Recuperado de http://doi.org/10.1016/j. artmed.2013.11.008

Waller, L. A. y Gotway, C. A. (2004). Applied spatial statistics for public health data. Hoboken, N.J: John Wiley \& Sons.

Wongkoon, S., Jaroensutasinee, M. y Jaroensutasinee, K. (2012). Development of temporal modeling for prediction of dengue infection in Northeastern Thailand. Asian Pacific Journal of Tropical Medicine, 5(3), 249-252. http://doi.org/10.1016/ S1995-7645(12)60034-0 